\title{
5-Aza-2-deoxycytidine Enhances the Sensitivity of 5-Fluorouracil by Demethylation of the Thymidine Phosphorylase Promoter
}

\author{
YUKIHIKO NISHIZAWA ${ }^{1}$, RYUJI IKEDA ${ }^{1,2}$, MASATATSU YAMAMOTO ${ }^{3}$, KOHICHI KAWAHARA ${ }^{3}$, \\ YOSHINARI SHINSATO ${ }^{3}$, KENTARO MINAMI ${ }^{3}$, MINA NITTA $^{1}$, HIDEYUKI TERAZONO $^{1}$, \\ SHIN-ICHI AKIYAMA ${ }^{3}$, TATSUHIKO FURUKAWA ${ }^{3,4}$ and YASUO TAKEDA ${ }^{1}$ \\ ${ }^{1}$ Department of Clinical Pharmacy and Pharmacology, Graduate School of Medical and Dental Sciences, \\ Kagoshima University, Kagoshima, Japan; \\ ${ }^{2}$ Department of Pharmacy, University of Miyazaki Hospital, Miyazaki, Japan; \\ ${ }^{3}$ Department of Molecular Oncology, Graduate School of Medical and Dental Sciences, \\ Kagoshima University, Kagoshima, Japan; \\ ${ }^{4}$ Center for the Research of Advanced Diagnosis and Therapy of Cancer, \\ Graduate School of Medical and Dental Sciences, Kagoshima University, Kagoshima, Japan
}

\begin{abstract}
Background/Aim: 5-Aza-2-deoxycytidine (5-Aza$C d R$ ) enhances the sensitivity to 5-fluorouracil (5-FU), but the molecular mechanism is not fully understood. The aim of this study was to investigate the molecular mechanism that enhances the sensitivity to 5-FU treated with 5-Aza-CdR via thymidine phosphorylase (TP). Materials and Methods: The sensitivity to drugs was determined on several cancer cell lines by the MTT assay. Protein and mRNA levels were examined by immunoblot and RT-PCR, respectively. Gene silencing, binding of Sp1 to DNA and methylation of DNA was performed by siRNA, ChIP assay and sodium bisulfate genomic sequencing, respectively. Results: Sp1-binding sites in the TP promoter were methylated in epidermoid carcinoma. 5-Aza-CdR demethylated Sp1-binding sites and enhanced sensitivity to 5-FU. Conclusion: Demethylation of Sp1-binding sites by 5-Aza-CdR was a key factor enhancing 5-FU sensitivity, which may enable more effective treatments for cancer patients with the combination of 5-Aza-CdR and 5-FU.
\end{abstract}

5-Fluorouracil (5-FU)-based regimens are extensively used to treat numerous solid tumors. 5-FU has an antitumor activity by inhibiting thymidylate synthase (TS), thus is a rate-limiting enzyme in DNA synthesis (1). First, 5-FU is

Correspondence to: Yasuo Takeda, Sakuragaoka 8-35-1, Kagoshima 890-8520, Japan. Tel: +81 992755543, Fax: +81 992655293, e-mail: takeda@m.kufm.kagoshima-u.ac.jp

Key Words: 5-fluorouracil, 5-aza-2-deoxycytidine, thymidine phosphorylase, methylation. converted by thymidine phosphorylase (TP) to 5-fluoro-2'deoxyuridine (FdUrd) (2) that is further converted by thymidine kinase to 5-fluoro-2'-deoxyuridine 5'monophosphate (FdUMP). FdUMP can also be derived from 5-fluoro-2'-deoxyuridine 5'-diphospihate, which is formed by the reductive synthesis of 5-fluoro-2'-deoxyuridine 5'diphospihate, a reaction catalyzed by ribonucleotide reductase. FdUMP binds to both TS and 5,10methylenetetrahdrofolate (CH2THF) leading to formation of a ternary complex that inhibits TS activities $(3,4)$. FdUMP can also be phosphorylated to 5-fluoro-2'-deoxyuridine 5'triphosphate, which can be misincorporated into DNA. Alternatively, 5-FU may be converted to 5-fluorouridine 5'triphosphate (FUTP), which can be incorporated into RNA and inhibit protein synthesis (1).

TP is also a platelet-derived endothelial cell growth factor (PD-ECGF) and a gliostatin (2). TP catalyzes the reversible phosphorolysis of thymidine, deoxyuridine, and their analogues to their respective bases and 2-deoxyribose-1phosphate $(5,6)$. Deoxyribosyltransferase converts a deoxynucleotide to another base to form a second deoxynucleoside. TP also activates some pyrimidine antimetabolites. The transcription region of TP has various transcription factor binding sites, including seven SP1 binding sites. In addition, it is known that the TP promoter region includes regions such as a interferon-stimulated response element (ISRE), and a gamma-activated sequence (GAS) which are transcription factor binding sites $(7,8)$. Sp1 contributes to the expression of TP in human cancer cells (9).

DNA methylation is known as an epigenetic modification, which takes place at cytosine residues in $\mathrm{CpG}$ dinucleotides, 
and causes long-term inactivation of transcription (10). Epigenetic drugs, such as the DNA methyltransferase inhibitor 5-aza-2'-deoxycytidine (5-Aza-CdR, decitabine), demethylates DNA and allows expression of silenced genes $(11,12)$. Mechanistically, 5-Aza-CdR, a nucleoside analog, is incorporated into the DNA of actively proliferating cells and irreversibly traps DNA methyltransferases (DNMTs) by forming covalent complexes $(13,14)$.

5 -Aza-CdR enhances the chemosensitivity of 5-FU in several cancer cells (15). However, the molecular basis of increased cancer cell sensitivity to 5 -FU by 5 -Aza-CdR is not fully understood. In this study, we examined the promoter region of thymidine phosphorylase and investigated the antitumor effect of 5-Aza-CdR in combination with 5-FU.

\section{Materials and Methods}

Reagents and antibodies. Monoclonal anti-TP antibody was used as described previously (16). Mouse anti-tubulin monoclonal antibody (DM1A) was obtained from Calbiochem (Merck, DS, Germany). Rabbit anti-Sp1 polyclonal antibody (PEP 2) was obtained from Santa Cruz Biotechnology (Santa Cruz, CA, USA). 5-Aza-CdR and 3-(4,5-dimethylthiazol-2-yl)-2,5-diphenyltetrazolium bromide (MTT) were obtained from Sigma Chemical Co (St. Louis, MO, USA). 5-FU and TPI, an inhibitor of thymidine phosphorylase, were obtained from Taiho Pharmaceutical Co.

Cell lines. Human lung cancer A549 cells, human epidermoid carcinoma KB3-1 cells, human breast ductal carcinoma MCF7 cells, and human uterine cervical carcinoma Yumoto cell were maintained in RPMI-1640 containing $10 \%$ fetal calf serum $1 \%$ PenicillinStreptomycin.

Quantification of drug sensitivity. The sensitivity of cells to drugs was determined by an MTT colorimetric assay as described previously (17).

Immunoblot analysis. According to the method of Laemmli (16), cellular proteins in the cells were separated using sodium dodecyl sulfate-polyacrylamide gel electrophoresis (SDS-PAGE), and then, proteins were transferred to a PVDF membrane. The transferred proteins were reacted with primary antibodies against TP, tubulin, or Sp1. After overnight incubation at $4^{\circ} \mathrm{C}$, membranes were washed with buffer 3 times and then incubated with HRP conjugated antimouse or anti-rabbit secondary antibodies (Cell Signaling Technology, MA, USA). After that, the target proteins were detected by e using an enhanced chemiluminescence western blotting detection system (GE healthcare., Buckinghamshire, UK).

RT-PCR methods. Total RNA extraction with TRIzol reagent and RT-PCR with SuperScript One-Step RT-PCR system were performed according instruction's manual. Briefly, Reaction mixtures contained $500 \mathrm{ng}$ total RNA, $0.2 \mathrm{mM}$ dNTPs, $0.2 \mu \mathrm{M}$ of each primer, enzyme mixture including SuperScript II RT, platinum Taq DNA polymerase, and 1x buffer with $1.2 \mathrm{mM} \mathrm{MgSO} 4$. RT procedure was followed: $50^{\circ} \mathrm{C}$ for $20 \mathrm{~min}$ and then at $94^{\circ} \mathrm{C}$ for 2 min. After that, PCR was performed as follows: 29 cycles at $94^{\circ} \mathrm{C}$
Table I. The IC $\mathrm{C}_{50}$ for 5-FU in KB3-1 cells treated with 5-FU in combination with or without 5-Aza-CdR or TPI.

\begin{tabular}{lcc}
\hline Drug sensitivity $\left[\mathrm{IC}_{50}(\mu \mathrm{M})\right]$ & \\
\hline Drugs & 5-Aza-CdR $(-)$ & 5-Aza-CdR $(500 \mathrm{nM})$ \\
TPI $(-)$ & $19.147 \pm 1.334$ & $3.802 \pm 1.584$ \\
TPI $(300 \mu \mathrm{M})$ & $21.958 \pm 1.138$ & $20.383 \pm 0.622$ \\
\hline
\end{tabular}

The sensitivity of KB3-1 cells to 5-FU with or without 5-Aza-CdR or TPI was determined by an MTT assay. Each value represents the mean \pm S.E. of three independent experiments.

for $15 \mathrm{sec}, 54^{\circ} \mathrm{C}$ for $30 \mathrm{sec}$, and $70^{\circ} \mathrm{C}$ for $30 \mathrm{sec}$. The primers for RT-PCR were designed based on human sequences in GenBank and were: TP, 5'-GCCAGAGATGTGACAGCCACCG-3' (sense), and 5'-TGGTGACCAGGTCCCTTAAGTC-3' (antisense); GAPDH, 5'AGAACATCATCCCTGCCTCTACTGG-3' (sense) and 5'AAAGGTGGAGGAGTGGGTGTCGCTG-3' (antisense).

Chromatin immunoprecipitation (ChIP) assays. ChIP assays were performed using a ChIP Assay Kit (EMD Millipore Corporation, Billerica, MA, USA). In brief, $5 \times 10^{5} \mathrm{~KB} 3-1$ and Yumoto cells were treated with or without $500 \mathrm{nM} 5$-Aza-CdR for $72 \mathrm{~h}$ and then harvested. The cells were cross-linked with formaldehyde (final concentration $1 \%$ ) and incubated for $10 \mathrm{~min}$ at $37^{\circ} \mathrm{C}$. Cells were then washed with ice-cold PBS containing protease inhibitors. Samples were lysed for $10 \mathrm{~min}$ in lysis buffer on ice, and Shearing of chromatin was performed by 10 cycles of sonication for $10 \mathrm{sec}$ and incubation on ice for 10 seconds. Cross-linked and released chromatin fractions were immunoprecipitated with magnetic beads and $\mathrm{Sp} 1$ antibodies, or non-specific IgG (rabbit) on a rolling shaker overnight at $4^{\circ} \mathrm{C}$. Cross-linking of the immunoprecipitates containing fragmented DNA was chemically reversed, and PCR was performed with KOD plus (TOYOBO, Osaka, Japan). The PCR primers used for amplifying promoters containing the Sp1-binding site were 5'-AACTGTGGGCCTTCCCACTC-3' (primer set 1 sense) and 5'-TGCTGAGGTCCTCGAAGAAAC-3' (primer set 1 antisense). Other primers for PCRs were designed based on human sequences in GenBank and were: primer set 2, 5' -CGTGTCCCAA CTCCGAACCCAGC-3' (sense), and 5'-ACACGCGGAACT TCCGGGCCGTC-3' (antisense); primer set 3, 5'-ACGGCCCGG AAGTTCCGCGTGTC-3' (sense), and 5'-TAGGGTTCAGGGT TCGCGCACAG-3' (antisense).

Assay for TP activity. TP activity was determined by a spectrophotometric method. KB3-1 cell lysates were incubated with $50 \mathrm{mM}$ potassium phosphate buffer $(\mathrm{pH} 6.5)$ and $10 \mathrm{mM}$ thymidine in a total volume of $0.1 \mathrm{ml}$. After $2 \mathrm{~h}$ incubation at $37^{\circ} \mathrm{C}$, the reaction was stopped by the addition of $1 \mathrm{ml} 0.2 \mathrm{~N} \mathrm{NaOH}$. Absorbance of the formed thymine was measured at $300 \mathrm{~nm}$.

Bisulphite conversion. Genomic DNA was isolated from KB3-1 cells, Yumoto cells, U937 cells, and Panc1 cells. DNA fragmentation was assessed on a $1 \%$ agarose gel by electrophoresis and quantified using a GeneQuuant pro (GE healthcare., Buckinghamshire, UK) for bisulphite conversion. The extracted DNA was subjected to bisulphite conversion using the Methylcode Bisulfite Conversion Kit according to instruction's manual (ThermoFisher Scientific Tokyo, 

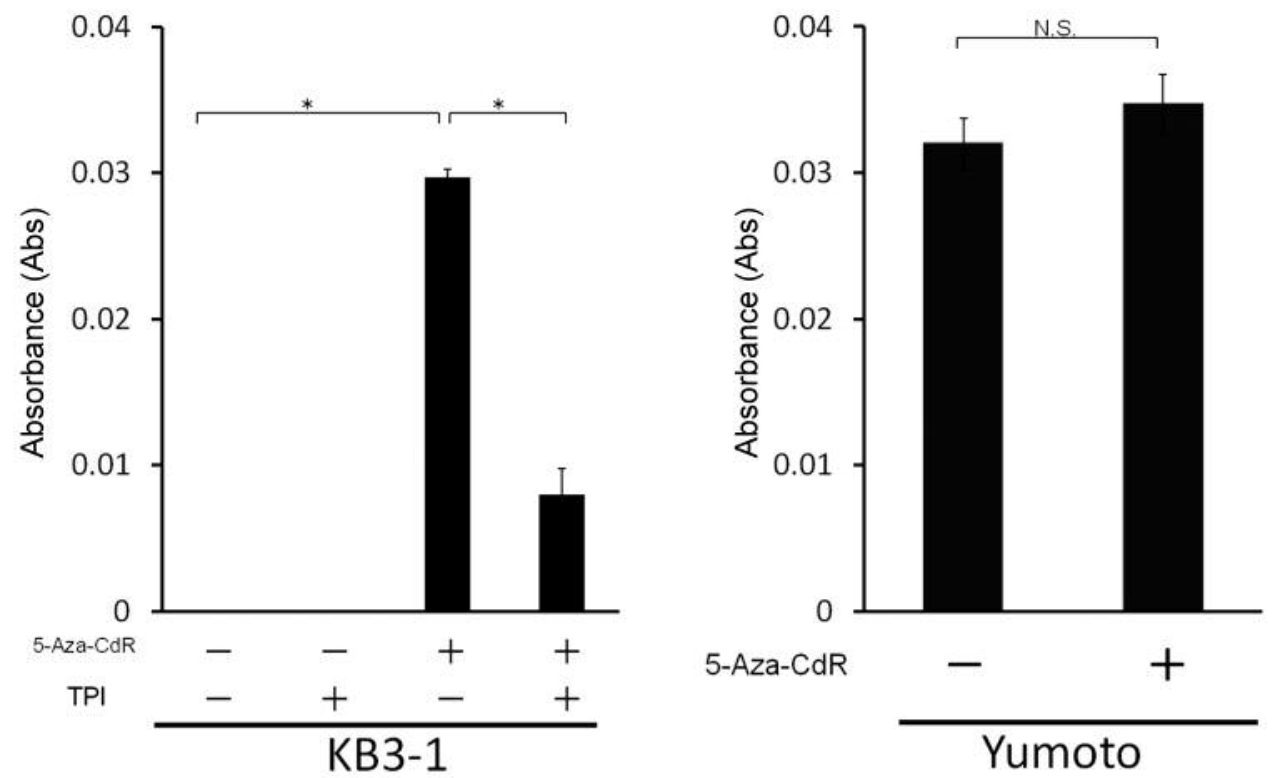

Figure 1. Enzymatic activity of TP in KB3-1 and Yumoto cells. TP activity in cell lysates was measured using thymidine as TP substrate. The effect

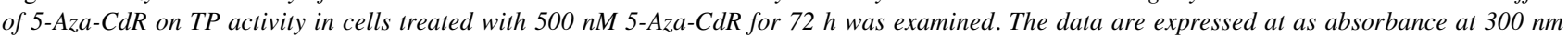
of the formed thymine. Statistical significance was determined by a Student's t-test. ${ }^{*} p<0.001 . n=4$.

Japan). Briefly, 500 ng of purified genomic DNA were mixed with CT Conversion Reagent. The reaction mixture was heated at $98^{\circ} \mathrm{C}$ for $10 \mathrm{~min}$ followed by incubation at $64^{\circ} \mathrm{C}$ for $2.5 \mathrm{~h}$ and further incubation at $4^{\circ} \mathrm{C}$ for up to $20 \mathrm{~h}$. Then, the mixture washed using a spin culmn system followed by desulphonation of the bisulphitetreated DNA. The desulphonation buffer was removed as flow through by centrifugation at $10,000 \mathrm{rpm}$ for $30 \mathrm{sec}$. After washing, the bisulphite-converted DNA was eluted with $20 \mu$ l of elution buffer. Eluted DNA was processed for bisulphite sequencing or stored at $-20^{\circ} \mathrm{C}$.

Bisulphite sequencing. The TP promoter region was amplified by PCR. The reaction mixture contained $100 \mathrm{nM}$ of each primer, 100 ng of bisulphite-converted DNA, 1.25U of TaKaRa EpiTaq HS DNA Polymerase (TaKaRa, Ohtsu, Japan), 10× EpiTaq PCR Buffer (Mg2+ free), $25 \mathrm{mM} \mathrm{MgCl} 2$, and dNTP mixture in a final volume of $50 \mu \mathrm{l}$. The PCR products were cloned using pBluescript SK (+) and a Luria-Bertani (LB) ampicillin plate. True positives were screened by colony PCR method using the M13 primer pair. Plasmid DNA was extracted using a miniprep kit (Qiagen, Hilden, Germany). The purified plasmid DNA for Bisulphite Sequencing was sequenced.

\section{Results}

5-Aza-CdR increased the efficiency of 5-FU. 5-Aza-CdR has been shown to enhance the chemosensitivity of 5-FU in several cancer cells (12). To investigate the effects of 5-Aza$\mathrm{CdR}$ on the efficacy of 5-FU, we verified the sensitivity of KB3-1 cells to 5-FU with or without 5-Aza-CdR by an MTT assay (Table I).

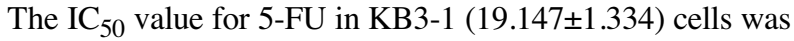
significantly higher than that in 5-Aza-CdR-treated KB3-1 cells (3.802 \pm 1.584$)$. TP expression is related to the sensitivity of cells to 5-FU (18). Therefore, we focused on TP and investigated the influence of TP on the sensitivity to 5-FU in cancer cells treated with 5-Aza-CdR using TPI, a TP enzymatic activity inhibitor. The $\mathrm{IC}_{50}$ value for 5-FU in $\mathrm{KB} 3-1$ cells treated with 5 -Aza-CdR in the presence of $500 \mathrm{nM}$ TPI was significantly higher than that in KB3-1 cells in the absence of TPI. These results suggested that the increased sensitivity to 5FU in cancer cells treated with $5-\mathrm{Aza}-\mathrm{CdR}$ required TP enzymatic activity.

5-Aza-CdR increased TP enzymatic activity and expression in some cancer cells. To determine whether 5-Aza-CdR affects TP enzymatic activity, TP enzymatic activity was examined in KB3-1 and Yumoto cells in the presence and absence of 5-AzaCdR (Figure 1). 5-Aza-CdR-treated KB3-1 cells had higher TP enzymatic activity than that of untreated cells. However, the induction of TP activity by 5-Aza-CdR was not detected in Yumoto cells. These results suggested that 5-Aza-CdR induced TP expression in KB3-1 cells but not in Yumoto cells.

Next, the effects of TP expression in cancer cells treated with 5 -Aza-CdR were evaluated by immunoblot analysis using an antibody against TP. As shown in Figure 2A, TP was highly expressed in Yumoto and MCF7 cells but at much lower levels in KB3-1 and A549 cells. When KB3-1 cells and A549 cells were treated with 5-Aza-CdR, the expression levels of TP 


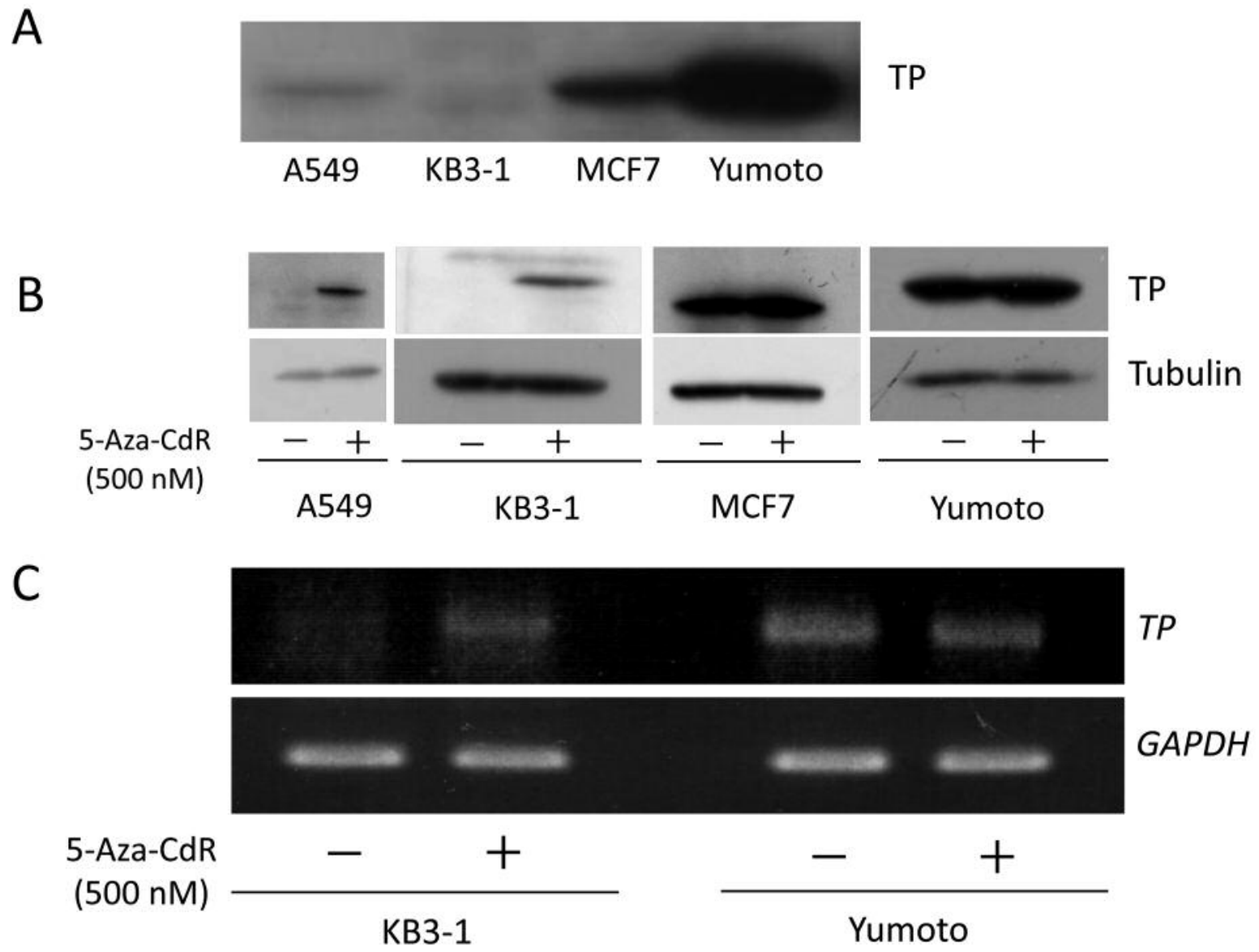

Figure 2. Effect of 5-Aza-CdR on the expression of TP in A549, KB3-1, MCF7, and Yumoto cells. (A) Lysates of A549, KB3-1, MCF7, and Yumoto cells were separated by SDS-PAGE and transferred to a PVDF membrane. TP cytosol levels (100 $\mu \mathrm{g}$ of protein) were determined by immunoblotting as described in materials and methods. (B) Expression of TP in KB3-1, MCF7, Yumoto, and U937 cells treated with and without 5-Aza-CdR. Cells were treated with or without 5-Aza-CdR at $500 \mathrm{nM}$ for $72 \mathrm{~h}$, and TP levels were determined by immunoblotting. (C) Expression of TP mRNA in KB3-1 and Yumoto cells treated with and without 5-Aza-CdR. Cells were treated with or without 5-Aza-CdR at $500 \mathrm{nM}$ for 72 h, and TP mRNA was detected using RT-PCR.

increased compared with the respective untreated cells. This induction of TP expression was not observed in MCF-7 cells and Yumoto cells (Figure 2B). The induction of TP mRNA in KB3-1 and Yumoto cells with 5-Aza-CdR was determined using RT-PCR. Treatment with 5-Aza-CdR increased TP mRNA levels in KB3-1 cells but not in Yumoto cells (Figure $2 \mathrm{C})$. These results indicated that 5-Aza-CdR enhanced TP transcription and increased $\mathrm{TP}$ protein expression and enzymatic activity in KB3-1 cells.

Spl knockdown decreased TP expression by 5-Aza-CdR. Because TP expression is regulated by $\mathrm{Sp} 1$ (19), we investigated whether TP induction by 5-Aza-CdR in KB3-1 cells depended on Sp1 expression. Sp1 knockdown using siRNA inhibited induction of TP expression by 5-Aza-CdR (Figure 3A), which indicated that TP induced by 5-Aza-CdR depended on Sp1 expression in KB3-1 cells.
Next, the levels of Sp1 and TP proteins were measured in KB3-1 cells treated with different 5-Aza-CdR concentrations. The levels of TP protein increased with increasing 5-Aza-CdR concentrations, but those of SP1 protein were not altered (Figure 3B).

5-Aza-CdR strengthened the bond between Spl and the TP promoter. The TP promoter region contains several Sp1binding elements (15). A schematic diagram of TP promoter region is shown in Figure 4A. Because TP induction by 5-AzaCdR depends on Sp1 expression, a ChIP assay was performed to determine which sequences in the TP promoter bind $\mathrm{Sp} 1$ and promote $T P$ transcription. In the presence of 5-Aza-CdR. a PCR product was detected only when using the primers F1 and R1 (Figure 4B), which suggests that Sp1 binds to the following sites of TP promoter: -1116 bp (Sp1-1116), -983 bp (Sp1-983), or -903 bp (Sp1-903). Sp1-binding sites on the TP promoter 

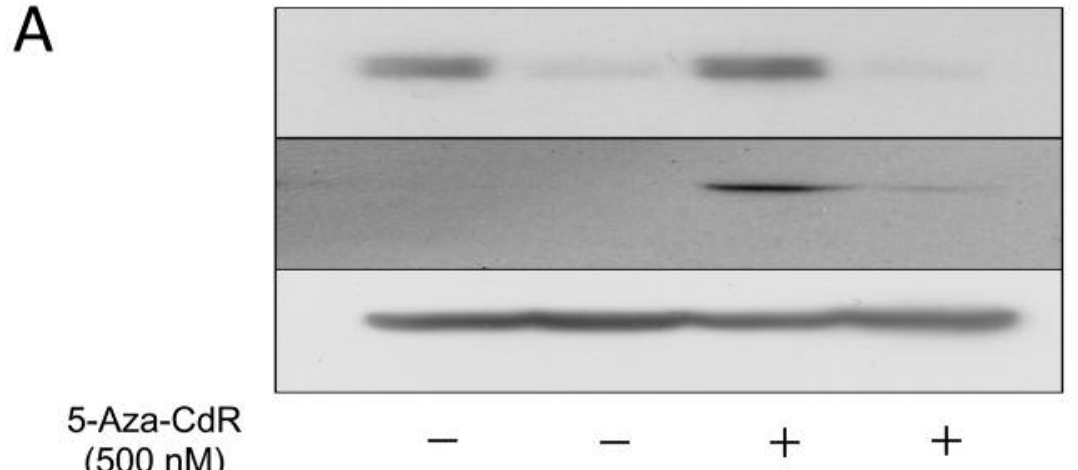

$\mathrm{Sp}-1$

TP

\section{5-Aza-CdR (500 nM) SiRNA}

\section{Control Sp-1 Control Sp-1}

\section{Tubulin}

B

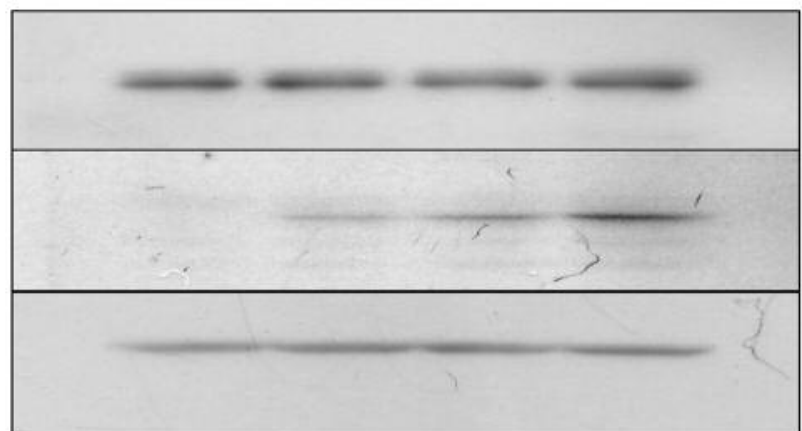

$\mathrm{Sp}-1$

TP

Tubulin

5-Aza-CdR

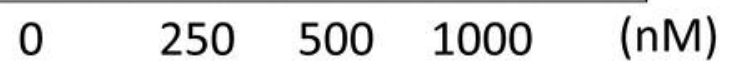

Figure 3. Effect of 5-Aza-CdR and Sp1-targeting siRNA on the expression of Sp1 in KB3-1 cells treated with 5-Aza-CdR. (A) Cells were transfected with Sp1-targeting or control siRNA. After a $24 \mathrm{~h}$ incubation and medium change, cells were treated with $500 \mathrm{nM} 5$-Aza-CdR for 72 h. Cell lysates were prepared, and the expression of Sp1 and TP was detected by immunoblotting. (B) The expression levels of TP and Sp1 in KB3-1 cells. The cells were treated with 5-Aza-CdR at the indicated concentrations for $72 \mathrm{~h}$, and the levels of TP and Spl were determined by immunoblotting.

Sp1-1116, Sp1-983, and Sp1-903 were consistent with Sp1dependent TP induction by 5-Aza-CdR.

Pattern and location of individual cytosine methylation sites in the TP promoter region. The methylation of Sp1-binding elements in the TP promoter region were examined by sodium bisulfate genomic sequencing. Yumoto cells, which express higher TP levels, lacked methylation in this region. By contrast, KB3-1 cells, which have no TP activity, had dense methylation at nearly every site examined throughout the entire promoter region and near Sp1-binding sites in the TP gene (data not shown). Sp1-1116, Sp1-983, and Sp1-903 were methylated in KB3-1 cells but not in Yumoto cells (Figure 5).

\section{Discussion}

5-FU has been used to treat diverse neoplastic diseases and is used in combination with other anticancer agents for various carcinomas. 5-Aza-CdR is a specific inhibitor of DNA methylation and functions similar to Azacytidine, but 5-AzaCdR can only be incorporated into DNA strands $(13,14)$.

5-Aza-CdR was found to increase the sensitivity of KB31 cells to 5-FU, and TPI abrogated the effects of 5-Aza-CdR. Furthermore, 5-Aza-CdR increased the enzymatic activity and expression of TP in KB3-1 cells but not in Yumoto cells. These results suggested that 5-Aza-CdR demethylates TP regulatory promoter regions and increases the sensitivity of KB3-1 cells to 5-FU.

In this study, we found that TP expression was regulated by epigenetic modifications. Inactivation of TP was associated with hypermethylation of $\mathrm{CpG}$ dinucleotides located in the TP promoter. We also found that Sp1-binding sites on the TP promoter are important for transcription. Ikuta et al. have reported that $\mathrm{Sp} 1$ transcription factor is essential for the expression of TP in rheumatoid fibroblastlike synoviocytes (19). The $T P$ promoter region has several potential Sp1-binding sites, an interferon-stimulated response element (ISRE), and a gamma-activated sequence $(7,8)$. 


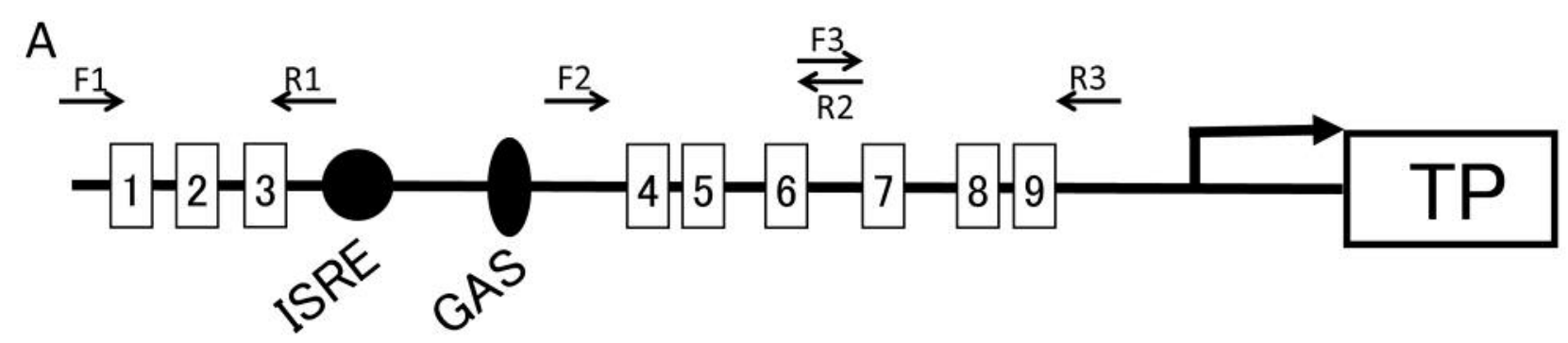

B IP: Sp-1

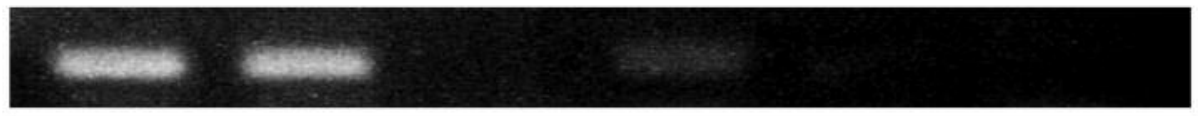

F1R1

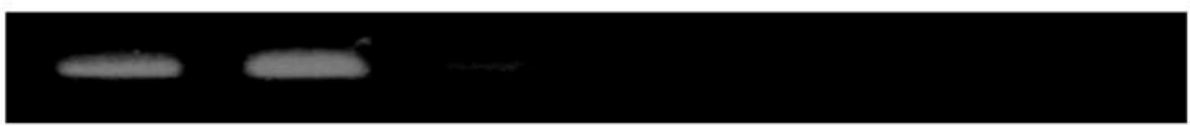

F2R2

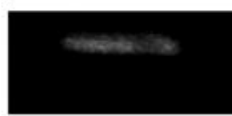

Conerans

$\sqrt{2}$

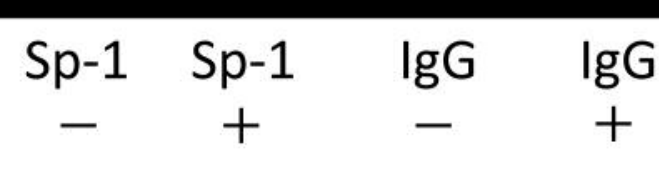

\section{Antibody \\ 5-Aza-CdR $(500 \mathrm{nM})$}

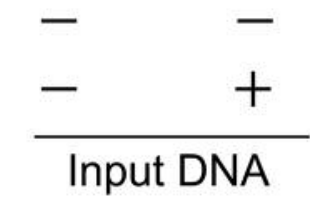

Figure 4. Effect of 5-Aza-CdR on Sp1 binding with ChIP assays. (A) Squares indicate each Sp1-binding site. A circle and an oval indicate the ISRE and GAS sequences, respectively. Arrows indicate the PCR primers, F1R1, F2R2, and F3R3, used for amplifying Sp1-binding sites in the TP promoter. (B) KB3-1 cells were incubated in the presence or absence of $500 \mathrm{nM} 5$-Aza-CdR for $72 \mathrm{~h}$. Spl binding to the specific sequences within the TP promoter was detected by ChIP assays.

ChIP assays showed that Sp1-1116, Sp1-983, and Sp1-903 have an important role in regulating the promoter activity of the TP gene (19).

Kosuri et al. have reported that methylation of extracellular growth factor-1 (ECGF-1) is a prediction marker of reduced efficacy of capecitabine (20). The DNA demethylating agent 5-Aza-CdR has synergistic effects on acute myeloid leukemia (AML) with the inhibitor of apoptosis (IAP) antagonist birinapant and on breast cancer cells with methyl donor S-adenosyl methionine (21). Kosuri et al. have also reported that the $\mathrm{CpG}$ islands of TP were methylated at a high frequency in mesothelioma patient tumor specimens (20). These results suggest that the TP gene is highly methylated in some patient tumors. Cancers have direct effects on the epigenome, chronic inflammation, viral infections, or microbiome changes, or indirect effects because of mutations in epigenetic-driver genes.

Several preclinical and clinical studies have demonstrated that intrinsic or acquired resistance to fluoropyrimidines correlates with low or deficient intra-tumoral TP expression. Therefore, elevation of TP enhances the sensitivity of tumor cells to fluoropyrimidines. A combination of fluoropyrimidines and several chemoagents, such as taxanes, cyclophosphamide, or EGFR tyrosine kinase inhibitors, have antitumor effects via TP up-regulation. Terranova-Barberio et al. have reported that the combined treatment with capecitabine and histone deacetylase inhibitors, such as valproic acid, resulted in a synergistic antiproliferative and pro-apoptotic effects in breast cancer cells but not in TP-knockout cells, which suggests that a combination of histone deacetylase inhibitors and capecitabine is an innovative antitumor strategy (22).

We previously reported the relationship between FdUMP and TP (2). The expression levels of FdUMP in TPoverexpressing cells were significantly higher than in parental cells. TPI significantly decreased the levels of FdUMP. The expression of early growth response protein-1 (Egr-1) and the angiogenesis inhibitor thrombospondin-1 (TSP1) were increased by 5 -FU in $\mathrm{KB} / \mathrm{TP}$ cells but only slightly in $\mathrm{KB} / \mathrm{CV}$ cells. These results indicated that the expression levels of TP in $\mathrm{KB} / \mathrm{CV}$ cells were low. TPI attenuated the induction of Egr-1 and TSP1 mRNA by 5-FU, and LV in the presence of 5-FU increased the expression of Egr-1 and TSP1 mRNA in $\mathrm{KB} / \mathrm{TP}$ cells. These findings suggested that TP has a role in the production of FdUMP and enhances the response to 5-FU in 

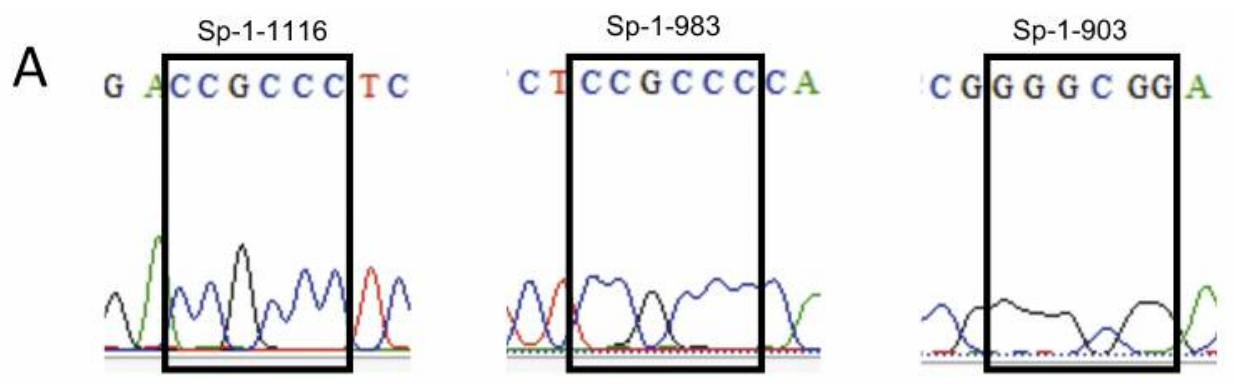

Bisulfit (-) 5-Aza-CdR(-)
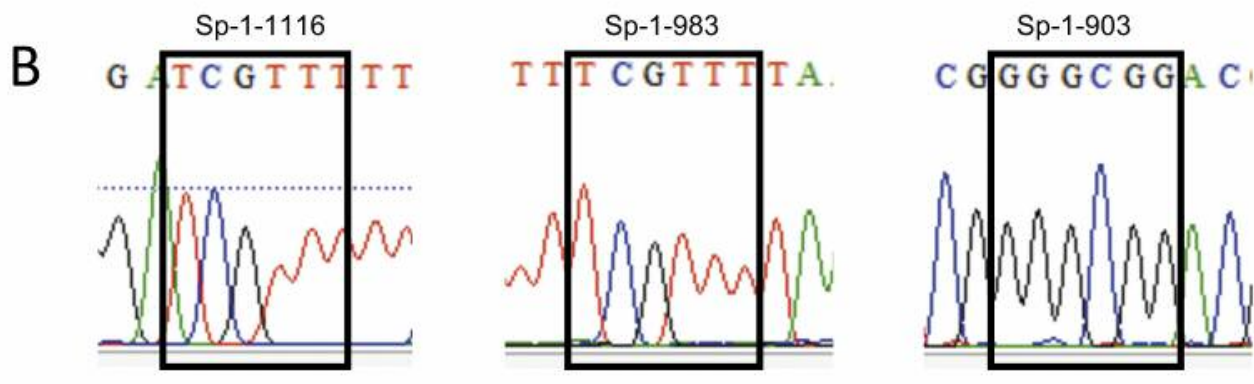

Bisulfit (+) 5-Aza-CdR(-)
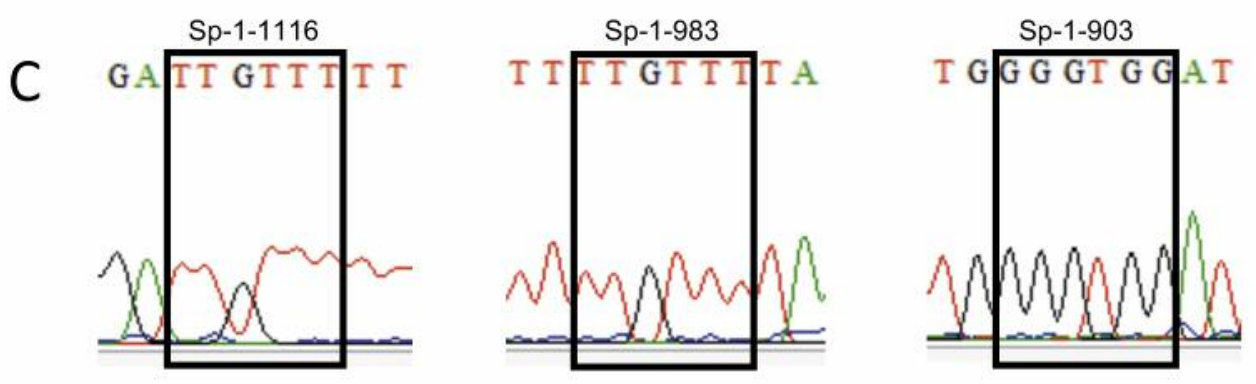

Bisulfit (+) 5-Aza-CdR(+)
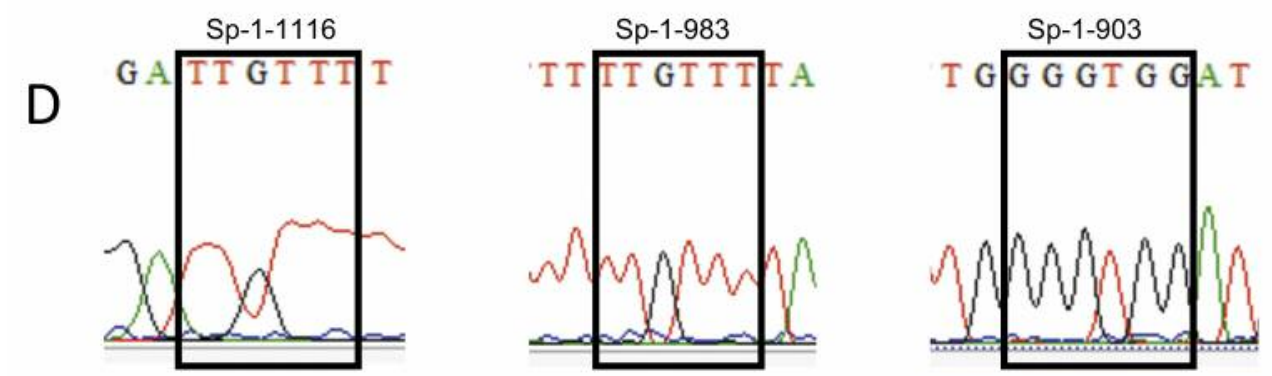

Bisulfit (+) 5-Aza-CdR(-)

Figure 5. Methylation of the Sp1-binding sites. Sequencing of the TP promoter region containing the Sp1-binding sites (Sp1-1116, Sp1-983, and Sp1903) for (A) genomic DNA of KB3-1 cells, (B) bisulfate-treated genomic DNA of KB3-1 cells, (C) bisulfate-treated genomic DNA of KB3-1 cells incubated with $500 \mathrm{nM}$ 5-Aza-CdR for $72 \mathrm{~h}$, and (D) bisulfate-treated genomic DNA of Yumoto cells. The boxed area indicates the specific Sp1-binding site.

cells with high expression of TP, and that FdUMP and FUTP induce Egr-1 and TSP1 in KB cells (2).

In summary, 5-Aza-CdR potentiated the anticancer activity of 5-FU by inducing thymidine phosphorylase expression.

\section{Conflicts of Interest}

The Authors have no conflicts of interests to declare regarding this study.

\section{Authors' Contributions}

YN and RI designed the study, and wrote the draft of the manuscript. MY and KK contributed to design and construct plasmids and assisted analysis. YS and KM contributed to subclone the plasmids and assisted in sample preparation. MN, HT and YT contributed to analysis and interpretation of data, and assisted in the preparation of the manuscript. SA, TF and YT critically reviewed the manuscript. 


\section{Acknowledgements}

The Authors would like to thank Katsushi Yamada for his support and advice at Kagoshima University.

\section{References}

1 Longley DB, Harkin DP and Johnston PG: 5-fluorouracil: Mechanisms of action and clinical strategies. Nat Rev Cancer 3(5): 330-338, 2003. PMID: 12724731. DOI: 10.1038/nrc1074

2 Matsushita S, Ikeda R, Nishizawa Y, Che XF, Furukawa T, Miyadera K, Tabata S, Ushiyama M, Tajitsu Y, Yamamoto M, Takeda Y, Minami K, Mataki H, Kanzaki T, Yamada K, Kanekura $\mathrm{T}$ and Akiyama $\mathrm{S}$ : The role of thymidine phosphorylase in the induction of early growth response protein1 and thrombospondin-1 by 5-fluorouracil in human cancer carcinoma cells. Int J Oncol 36(5): 1193-1200, 2010. PMID: 20372793. DOI: 10.3892/ijo_00000602

3 Santi DV, McHenry CS and Sommer H: Mechanism of interaction of thymidylate synthetase with 5-fluorodeoxyuridylate. Biochemistry 13(3): 471-481, 1974. PMID: 4203910. DOI: $10.1021 / \mathrm{bi00700a012}$

4 Sommer H and Santi DV: Purification and amino acid analysis of an active site peptide from thymidylate synthetase containing covalently bound 5-fluoro-2'-deoxyuridylate and methylenetetrahydrofolate. Biochem Biophys Res Commun 57(3): 689-695, 1974. PMID: 4275130. DOI: 10.1016/0006-291x(74)90601-9

5 Iltzsch $\mathrm{MH}$, el Kouni $\mathrm{MH}$ and Cha S: Kinetic studies of thymidine phosphorylase from mouse liver. Biochemistry 24(24): 6799-6807, 1985. PMID: 4074727. DOI: 10.1021/ bi00345a011

6 Friedkin M and Roberts D: The enzymatic synthesis of nucleosides. I. Thymidine phosphorylase in mammalian tissue. J Biol Chem 207(1): 245-256, 1954. PMID: 13152099.

7 Hagiwara K, Stenman G, Honda H, Sahlin P, Andersson A, Miyazono K, Heldin CH, Ishikawa F and Takaku F: Organization and chromosomal localization of the human platelet-derived endothelial cell growth factor gene. Mol Cell Biol 11(4): 21252132, 1991. PMID: 2005900. DOI: 10.1128/ mcb.11.4.2125

8 Goto H, Kohno K, Sone S, Akiyama S, Kuwano M and Ono M: Interferon gamma-dependent induction of thymidine phosphorylase/platelet-derived endothelial growth factor through gamma-activated sequence-like element in human macrophages. Cancer Res 61(2): 469-473, 2001. PMID: 11212233.

9 Zhu GH, Lenzi M and Schwartz EL: The sp1 transcription factor contributes to the tumor necrosis factor-induced expression of the angiogenic factor thymidine phosphorylase in human colon carcinoma cells. Oncogene 21(55): 8477-8485, 2002. PMID: 12466967. DOI: 10.1038/sj.onc. 1206030

10 Holliday R and Pugh JE: DNA modification mechanisms and gene activity during development. Science 187(4173): 226-232, 1975. PMID: 1111098.

11 Egger G, Liang G, Aparicio A and Jones PA: Epigenetics in human disease and prospects for epigenetic therapy. Nature 429(6990): 457-463, 2004. PMID: 15164071. DOI: 10.1038/ nature 02625
12 Yoo CB and Jones PA: Epigenetic therapy of cancer: Past, present and future. Nat Rev Drug Discov 5(1): 37-50, 2006. PMID: 16485345 . DOI: $10.1038 / \mathrm{nrd} 1930$

13 Santi DV, Garrett CE and Barr PJ: On the mechanism of inhibition of DNA-cytosine methyltransferases by cytosine analogs. Cell 33(1): 9-10, 1983. PMID: 6205762.

14 Santi DV, Norment A and Garrett CE: Covalent bond formation between a DNA-cytosine methyltransferase and DNA containing 5-azacytosine. Proc Natl Acad Sci USA 81(22): 6993-6997, 1984. PMID: 6209710. DOI: 10.1073/pnas.81.22.6993

15 Mirza S, Sharma G, Pandya P and Ralhan R: Demethylating agent 5-aza-2-deoxycytidine enhances susceptibility of breast cancer cells to anticancer agents. Mol Cell Biochem 342(1-2): 101-109, 2010. PMID: 20455010. DOI: 10.1007/s11010-0100473-y

16 Takebayashi Y, Akiyama S, Akiba S, Yamada K, Miyadera K, Sumizawa T, Yamada Y, Murata F and Aikou T: Clinicopathologic and prognostic significance of an angiogenic factor, thymidine phosphorylase, in human colorectal carcinoma. J Natl Cancer Inst 88(16): 1110-1117, 1996. PMID: 8757190. DOI: $10.1093 /$ jnci/88.16.1110

17 Carmichael J, DeGraff WG, Gazdar AF, Minna JD and Mitchell JB: Evaluation of a tetrazolium-based semiautomated colorimetric assay: Assessment of chemosensitivity testing. Cancer Res 47(4): 936-942, 1987. PMID: 3802100.

18 Haraguchi M, Furukawa T, Sumizawa T and Akiyama S: Sensitivity of human $\mathrm{kb}$ cells expressing platelet-derived endothelial cell growth factor to pyrimidine antimetabolites. Cancer Res 53(23): 5680-5682, 1993. PMID: 7694792.

19 Ikuta K, Waguri-Nagaya Y, Kikuchi K, Yamagami T, Nozaki M, Aoyama M, Asai K and Otsuka T: The sp1 transcription factor is essential for the expression of gliostatin/thymidine phosphorylase in rheumatoid fibroblast-like synoviocytes. Arthritis Res Ther 14(2): R87, 2012. PMID: 22534375. DOI: 10.1186/ar3811

20 Kosuri KV, Wu X, Wang L, Villalona-Calero MA and Otterson GA: An epigenetic mechanism for capecitabine resistance in mesothelioma. Biochem Biophys Res Commun 391(3): 14651470, 2010. PMID: 20035722 DOI: 10.1016/j.bbrc.2009.12.095

21 Yen CY, Huang HW, Shu CW, Hou MF, Yuan SS, Wang HR, Chang YT, Farooqi AA, Tang JY and Chang HW: DNA methylation, histone acetylation and methylation of epigenetic modifications as a therapeutic approach for cancers. Cancer Lett 373(2): 185-192, 2016. PMID: 26808576. DOI: 10.1016/ j.canlet.2016.01.036

22 Terranova-Barberio M, Roca MS, Zotti AI, Leone A, Bruzzese F, Vitagliano C, Scogliamiglio G, Russo D, D’Angelo G, Franco R, Budillon A and Di Gennaro E: Valproic acid potentiates the anticancer activity of capecitabine in vitro and in vivo in breast cancer models via induction of thymidine phosphorylase expression. Oncotarget 7(7): 7715-7731, 2016. PMID: 26735339. DOI: $10.18632 /$ oncotarget.6802

Received April 4, 2019

Revised June 27, 2019

Accepted June 28, 2019 